\title{
Multi-Modal Processing of Graphene Towards Precisely Controlled Fabrication of a Nanoelectronic Device Using the Helium Ion Microscope and the TOF SIMS
}

Songkil Kim ${ }^{1,2}$, Anton V. Ievlev ${ }^{1,2}$, Ivan V. Vlassiouk ${ }^{3}$, Matthew J. Burch ${ }^{1,2}$, Xiahan Sang ${ }^{1,2}$, Chance Brown $^{1,4}$, Raymond R. Unocic ${ }^{1,2}$, Alex Belianinov ${ }^{1,2}$, Stephen Jesse ${ }^{1,2}$ and Olga S. Ovchinnikova ${ }^{1,2}$

1. Center for Nanophase Materials Sciences, Oak Ridge National Laboratory, Oak Ridge, TN 37831, USA

2. Institute for Functional Imaging of Materials, Oak Ridge National Laboratory, Oak Ridge, TN 37831, USA

3. Energy \& Transportation Science Division, Oak Ridge National Laboratory, Oak Ridge, TN 37831, USA

4. Bredesen Center, University of Tennessee, Knoxville, TN 37996, USA.

Graphene has been under intense exploration as an electronic material owing to its excellent electronic, mechanical and thermal properties. [1] The two-dimensional aspect of this material allows controlled modification of its properties, which can be utilized to design new functional devices. [2-4] However, there are challenges in fabrication of graphene nanodevices, due to surface contamination and difficulties in controlled patterning and chemical functionalization of graphene. To overcome these challenges, we have developed graphene processing protocols using the Time-of-Flight Secondary Ion Mass Spectrometry (ToF-SIMS) combined with Atomic Force Microscopy (AFM) and the helium ion microscope (HIM) for surface cleaning and direct-write patterning.

To clean graphene on $\mathrm{a} \mathrm{SiO}_{2} / \mathrm{Si}$ substrate, we used an Argon molecular cluster beam with the size of $\sim 990$ atoms (current $\sim 4 \mathrm{nA}$ and spot size $\sim 20 \mu \mathrm{m}$ ) accelerated at $2.5 \mathrm{keV}$. Figures 1(a) and 1(b) demonstrate the surface before and after Ar sputtering. Figure 1(a) shows an AFM tapping mode topography image of the graphene surface with 1-2 nm thickness PMMA residues left after the graphene transfer from $\mathrm{Cu}$ onto the $\mathrm{SiO}_{2} / \mathrm{Si}$ substrate. Figure 1(b) is the AFM measured surface after 13 seconds of Ar sputtering over an area of $300 \mu \mathrm{m} \times 300 \mu \mathrm{m}$. Figure 1(c) illustrates the depth profiles of ionic species measured by the ToF-SIMS to control a sputtering process. Rapid decrease of hydrocarbon species $\left(\mathrm{C}_{2} \mathrm{H}^{-}\right.$and $\left.\mathrm{CH}^{-}\right)$from the beginning of the sputtering process to about $40 \mathrm{~s}$ was found and correlated with sputtering of the PMMA residues from the surface of graphene. After about $40 \mathrm{~s}$, the intensity of oxygen gradually increases, indicating the exposure of the $\mathrm{SiO}_{2}$ surface due to etching of the graphene areas. The in-situ monitoring of the sputtering process allowed us to establish a technique for precise control of the cleaning process to minimize the PMMA contamination along with minimization of the graphene damage.

After cleaning graphene surface by Ar irradiation, a graphene nanoelectronic device was fabricated using the HIM. Figure 2 shows two modes (milling and deposition) of HIM-based 'direct-write' nanofabrication of a simple graphene nanoelectronic device. Both fabrication modes utilize the same tool without any additional preparation steps, offering rapid fabrication of devices with high-resolution. Figure 2(a) is an array of holes milled in graphene, illustrating HIM resolution. Controlling beam parameters allowed us to mill holes down to the lateral size of $\sim 3 \mathrm{~nm}$. In particular, we can use this technique to fabricate a fully suspended graphene nanoelectronic device (Fig. 2b). To do this, graphene layer suspended on a TEM silicon nitride grid was patterned into a well-defined $20 \mathrm{~nm}$ wide strip. Then, three platinum/carbon electrodes, including source-drain contacts and a top-gate, were deposited with an assist of a gas injection system in the HIM chamber. Finally, two ends of the graphene strip were milled 
out to fabricate a fully suspended graphene channel separated from other graphene. This fabrication process produces high-resolution graphene patterning and electrode deposition without a 'halo' effect which can, otherwise, affect graphene's transport properties significantly. [3]

In conclusion, we illustrate a multi-modal processing workflow to clean and fabricate graphene nanoelectronic devices, using the ToF-SIMS and the HIM. We believe this level of chemical controls as well as nanometer precision of the fabrication process will enable the development of novel functional, molecular and nanodevices at improved production rates with fewer processing steps.

\section{References:}

[1] A K Geim and K S Novoselov, Nat. Mater. 6 (2007), 183-191.

[2] T Kuila et al, Prog. Mater Sci. 57 (2012), 1061-1106.

[3] S Kim et al, Nanoscale 7 (2015), 14946-14952.

[4] V Iberi et al, Sci. Rep. 5 (2015), 11952.

[5] This work was supported by the Oak Ridge National Laboratory's Center for Nanophase Materials Sciences (CNMS), which is a U.S. Department of Energy, Office of Science User Facility.

(a)

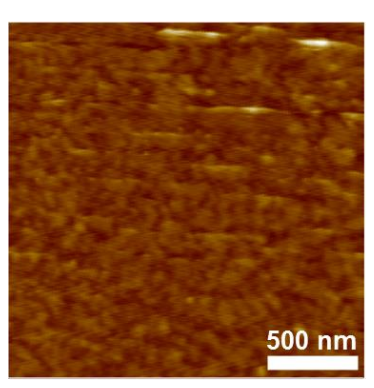

(b)

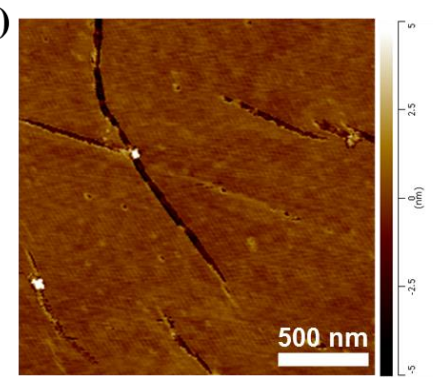

(c)

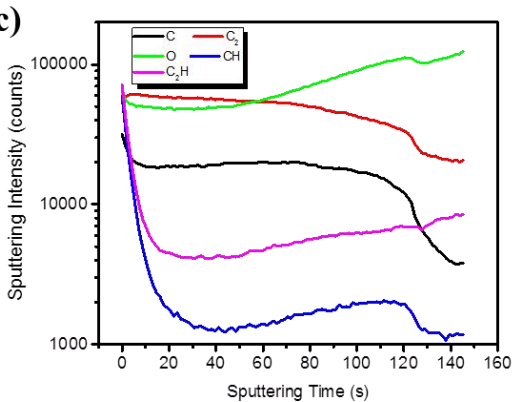

Figure 1. AFM tapping mode topography images of a graphene surface with PMMA residues (a) before and (b) after the surface cleaning for $13 \mathrm{~s}$ by the Ar cluster beam irradiation, and (b) the ToF SIMS depth profile of the graphene-PMMA surface supported by a $\mathrm{SiO}_{2} / \mathrm{Si}$ substrate.

(a)

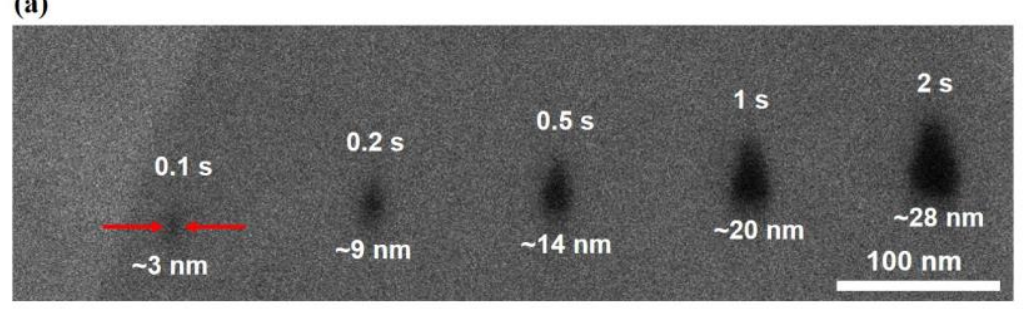

(b)

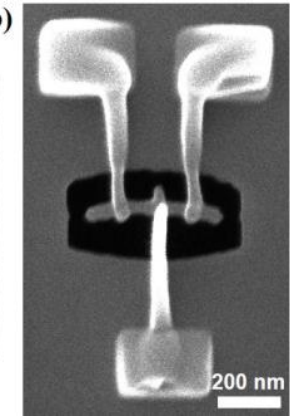

Figure 2. (a) Nanoholes milled in suspended graphene by helium ion beam irradiations (beam energy $25 \mathrm{keV}$ and current $1.5 \mathrm{pA}$ ) in a spot mode for different beam dwell time, and (b) a prototype of a suspended graphene nanoelectronic device fabricated by the two modes (milling and deposition) of the HIM-based nanofabrication. 